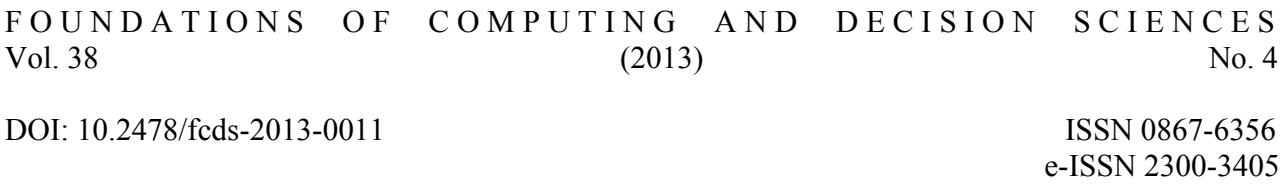

\title{
EOQ REVISITED WITH SUSTAINABILITY CONSIDERATIONS
}

\author{
M. Can ARSLAN and Metin TURKAY*
}

\begin{abstract}
The economic order quantity (EOQ) model is a pure economic model in classical inventory control theory. The model is designed to find the order quantity so as to minimize total cost under a deterministic setting. In this study, we revise the standard EOQ model to incorporate sustainability considerations that include environmental and social criteria in addition to the conventional economic considerations. We propose models for a number of different policies and analyze these revised models. Based on our analysis, we show how the triple bottom line considerations of sustainability be appended to traditional cost accounting in EOQ model. We also provide a number of useful insights for decision and policy making practices.
\end{abstract}

Keywords: supply chain management, economic order quantity model, inventory control, sustainability, operations management

\section{Introduction}

The economic order quantity (EOQ) model is a pure economic model in the classical inventory control theory. The model is designed to find the order quantity so as to minimize the total average cost of replenishment under deterministic demand conditions and assumptions (these assumptions are listed in section 2.1).

In this paper, we address the revision of this practical model to encompass the perspective of sustainability. We modify the standard model to further account for environmental and social criteria in addition to economic considerations. These added criteria stems from the emerging requirement of sustainability in supply chain management. The decision and policy makers are under pressure to incorporate these added dimensions into the decision making process as well as the conventional economics. This approach is referred to as the "triple bottom line accounting" [1].

We apply the triple bottom line accounting approach in a methodical manner. Our method is based on revising the standard EOQ model with additional objectives and/or constraints regarding those added criteria. We propose models for a number of different settings and analyze these revised models to characterize the optimal policy analytically and numerically. Based on our analysis, we show how these additional criteria can be

\footnotetext{
* Department of Industrial Engineering, Koc University, Rumelifeneri Yolu, 34450 Sariyer, Istanbul, Turkey. The first version of this paper was available to public on Dec. 2, 2010.
} 
appended to traditional cost accounting in order to achieve sustainability in supply chain management. We propose a number of useful insights for decision and policy makers.

\subsection{Related Literature}

Incorporating sustainability into the traditional supply chain and operations management problems has attracted limited attention in the open literature. Many review papers in this context are published during the last two decades including [2-12].

A significant part of the research in this area focuses only on the environmental aspects along with the economical objectives; social aspects are rarely included or completely ignored [11]. Furthermore, a methodical approach with a theoretical background in analyzing sustainable supply chain systems is also missing [11]. Our approach proposed in this paper is one of the first attempts in this direction in the open literature.

There are a number studies on the sustainability of supply chains. Bonney and Jaber [13] provide a list of non-cost metrics for incorporating environmental footprint in the inventory context and propose an environmentally enhanced EOQ model. Chen et al. [14] integrate carbon emission concerns into lot sizing models. The authors point out that existing literature in supply chain management lacks such carbon-enhanced operational models and provide insights based on their models and numerical experiments to show the effect of carbon accounting. Manikas and Godfrey [15] propose a newsvendor model which maximizes expected profit of a manufacturer in the presence of emission permits and penalties. Letmathe and Balakrishnan [16] present a linear and a mixed-integer program for firms to determine the optimal product mix and production quantities under environmental constraints in addition to the production constraints. Penkuhn et al. [17] present a constrained nonlinear program for a chemical process production planning problem. Bauer et al. [18] incorporate greenhouse gas emission considerations into freight transportation planning and propose a multi-commodity capacitated network design formulation with the objective of minimizing the amount of greenhouse gas emissions due to transportation activities. They provide a computational illustration on a real-life rail freight transportation problem. Kim et al. [19] provide a bi-criteria optimization model to examine the relationship between the freight transport costs and carbon dioxide emissions for intermodal and truck-only freight networks. Lee et al. [20] present deterministic and stochastic programming formulations for logistics network design which account for environmental impacts as well as costs. Chaabane et al. [21] propose a mathematical programming formulation for the entire life-cycle design of a supply chain which incorporates environmental objectives as well. Neto et al. [22] investigate the environmental impact factors along the supply chain and propose a multi objective programming based framework in modeling and optimizing the design of logistic networks. Erkut et al. [23] present a multi-criteria mixed-integer linear programming formulation to solve a locationallocation problem regarding municipal waste management.

To our knowledge, there is no paper available in the open literature that provides a revised EOQ model with environmental considerations as well as the social dimension of sustainability. We revise the classical EOQ model to account for added environmental and social dimensions of sustainability as well as the economic one. In section 2, we develop and analyze such models. We propose managerial insights based on our analysis in section 3. Finally we discuss the contributions and implications of our study in section 4 . 


\section{Model Formulations \& Analysis}

In this section we present the original and revised EOQ models to integrate the triple bottom line of sustainability. We first present the standard model, then incorporate five different environmental considerations, next present social considerations with two different considerations and finally present the last model to incorporate the triple bottom line of sustainability. We also provide analysis of each of these models.

\subsection{The Standard Economic Order Quantity (EOQ) Model}

The EOQ model arises from the simplest form of economies of scale [24]. The model assumes a single item at a single location with a continuous demand. The demand is known and has a constant rate, $\lambda$, over time. A supply of the required item is needed in order to satisfy the demand. Therefore, either the items are produced or an order to the supplier is placed. The model assumes constant lead times with an infinite supply capacity where stock-outs are not allowed. Under these assumptions, the EOQ problem is to decide on the ordered quantity $Q$ (or the quantity to be produced) which minimizes the total average cost of replenishment; i.e. we have a single decision variable, $Q$, which satisfies $Q^{*}=\operatorname{argmin}$ $\{C(Q)\}$ optimally; where $C(Q)$ denotes the total average cost of replenishment. $C(Q)$ has two components: replenishment cost and the inventory holding cost. The replenishment cost consists of purchasing (or production) cost and the ordering cost [25].

The inventory level at time $t$ is denoted by $I(t)$. The inventory level in the EOQ model is assumed to be cyclic, each cycle starting from the inventory level $I(t)=Q$ (assuming zero inventory at $t=0$ ) and gradually depleted until the end of the cycle with the constant demand rate $\lambda$. Therefore, we obtain an average inventory of $Q / 2$ at each cycle is obtained. This result holds for any time horizon of all cycles since the cycles are identical [25]. The cost per unit item held per unit time is given by $h$. Then the inventory holding cost in $C(Q)$ becomes $h Q / 2$. The fixed/setup cost of ordering/production and the variable cost per unit ordered/produced are given by $K$ and $c$ respectively. Then, the sum of purchasing and ordering/production costs becomes $K+c Q$ at each cycle. Since each cycle is of length $T=Q / \lambda$, the $C(Q)$ is given by,

$$
C(Q)=\frac{K+c Q}{Q / \lambda}+h^{Q} / 2=\frac{K \lambda}{Q}+\lambda c+\frac{h Q}{2}
$$

The optimal $Q^{*}$ is then found by solving $C^{\prime}(Q)=0$ and checking if $C^{\prime \prime}(Q)>0$ for convexity of the cost function. Since $C(Q)$ is convex, the first order condition ensures optimality. Therefore the economic order quantity or the optimal $Q$ to the above optimization problem is given by,

$$
Q^{*}=\sqrt{\frac{2 K \lambda}{h}}
$$

which is also known as the Wilson's or Harris' formula [25, 26].

The EOQ model account solely for the economics of the replenishment and inventory holding activity related to the provision of the required items. Apart from the economic 
aspects, these activities also have impacts on the environment and the society which should be considered as well, as elaborated in the next sections.

\subsection{EOQ Model with Environmental Criterion}

In this section, we revise the standard EOQ model by taking carbon footprint into account. A typical environmental effect caused by most industrial operations is the inevitable release of greenhouse gases (GHG) [27]. As a result, in order to assess the environmental performance of an organization, the amount of GHG emissions is commonly used in the green/environmentally conscious supply chain and operations management literature (see [27] and the references therein). The set of greenhouse gases (including carbon dioxide) released by an organization due to its operations is commonly referred to as the carbon footprint [28].

In this paper, we consider carbon footprint in modeling the environmental criterion. We observe costs, emissions, and the refined order/production quantities under a number of different settings. In the following sections, five environmental management approaches each with different characteristics are modeled and analyzed.

\subsubsection{Direct Accounting}

The first approach to model carbon footprint is to treat it as an additional source of economic cost. Let $f$ be the fixed cost of environmental impact for each replenishment cycle due to setups, order processing or transportation; $v$ be the variable cost of environmental impact due to the production and related activities, and finally $g$ be the cost of environmental impact due to the inventory holding as a result of material handling and warehousing activities (one can refer to [29-33] for carbon footprint measurement standards and methodologies).

With these additional parameters, we can express $C\left(Q_{e e}\right)$ as,

$$
C\left(Q_{e e}\right)=\frac{K+f+(c+v) Q_{e e}}{Q_{e e} / \lambda}+(h+g) \frac{Q_{e e}}{2}=\frac{(K+f) \lambda}{Q_{e e}}+\lambda(c+v)+\frac{(h+g) Q_{e e}}{2}
$$

Proposition 1. The optimal order/production quantity of the direct emissions accounting model is $Q_{e e}^{*}=\sqrt{\frac{2(K+f) \lambda}{(h+g)}}$ and the corresponding optimal total cost is $C\left(Q_{e e}^{*}\right)=\lambda(c+v)+$ $\sqrt{2(K+f) \lambda(h+g)}$. Moreover, the following set of relationships holds:

(i) $Q_{e e}^{*}=Q^{*} \Leftrightarrow \frac{K}{h}=\frac{f}{g}$

(ii) $Q_{e e}^{*}>Q^{*} \Leftrightarrow \frac{K}{h}<\frac{f}{g}$

(iii) $Q_{e e}^{*}<Q^{*} \Leftrightarrow \frac{K}{h}>\frac{f}{g}$

Proof. Inserting $K=K+f$ and $h=h+g$ in the the standard model and by a similar derivation, the optimal order/production lot size is found as $Q_{e e}^{*}=\sqrt{\frac{2(K+f) \lambda}{(h+g)}}$. Plugging $Q_{e e}^{*}$ into $C(\mathrm{Q})$ yields 
$C\left(Q_{e e}^{*}\right)=\frac{(K+f) \lambda}{\sqrt{\frac{2(K+f) \lambda}{(h+g)}}}+\lambda(c+v)+\frac{(h+g) \sqrt{\frac{2(K+f) \lambda}{(h+g)}}}{2}=\lambda(c+v)+\sqrt{2(K+f) \lambda(h+g)}$.

(i) $\Leftrightarrow)$ Assume that $Q_{e e}^{*}=\sqrt{\frac{2(K+f) \lambda}{(h+g)}}=\sqrt{\frac{2 K \lambda}{h}}=Q^{*}$. This implies that $\frac{K+f}{h+g}=\frac{K}{h}=\frac{f}{g} . \quad(\Leftarrow)$ Can be derived similarly as in $(\Rightarrow)$. (ii), (iii) Can be derived similarly as in part (i).

Hence, the optimal order/production quantity is governed by the trade-off between replenishment and inventory holding costs with the only change of added environmental cost components. The refined optimal order/production quantity may be larger or smaller than the EOQ as it might be equal to it as well depending on the values of the cost components.

$Q_{e e}^{*}$ resembles the usual EOQ apart from $f$ and $g$. If these added parameters are incorporated into $K$ and $h$, then $Q_{e e}^{*}$ reduces to EOQ exactly. Otherwise, one of the cases in Proposition 1 (i) - (iii) applies. The unit inventory holding cost, $h$, incorporates the opportunity cost of the capital committed in addition to the real costs of inventory holding. However, such an argument is clearly not valid for $g$. Hence, $g$ might be smaller than $h$ in most practical situations. On the other hand, fixed environmental impacts are induced at all stages of the production, transportation, and order processing activities. Hence $f$ might be larger than $K$. Therefore, Proposition 1 (ii) is more likely to be realized when the added environmental impact is considered; i.e. $Q_{e e}^{*}>Q^{*}$.

The direct accounting approach is an optional one and left to the discretion of the managers of the organization, i.e. organizations may or may not calculate total cost by using the environmental cost components. However, the above analysis shows that there is value for the organization in investigating the sources of emissions and the related costs.

\subsubsection{Carbon Tax}

Organizations may be given incentive to account for the environmental costs through an externally applied carbon tax by the regulatory agencies. A simple tax schedule is a linear one; i.e. organizations pay an amount of $p$ money-units for each unit of carbon emitted. However, other tax schedules (convex/progressive, concave/regressive, non-linear, piecewise linear, staircase etc.) may also be applied as well. We consider only the linear tax schedule in this paper. The refined model with a linear tax schedule is as follows:

$$
C(\mathrm{Q})=\frac{K+p f+(c+p v) Q}{Q / \lambda}+(h+p g) \frac{Q}{2}=\frac{(K+p f) \lambda}{Q}+\lambda(c+p v)+\frac{(h+p g) Q}{2}
$$

Proposition 2. The optimal order/production quantity of the carbon tax model is $Q_{e e}^{*}=$ $\sqrt{\frac{2(K+p f) \lambda}{(h+p g)}}$ and the corresponding optimal total cost is $C\left(Q_{e e}^{*}\right)=\lambda(c+p v)+\sqrt{2(K+p f) \lambda(h+p g)}$.

Proof. Inserting $K=K+p f$ and $h=h+p g$ in the the standard model and by a similar derivation, the optimal order/production lot size is found as $Q_{e e}^{*}=\sqrt{\frac{2(K+p f) \lambda}{(h+p g)}}$. Plugging $Q_{e e}^{*}$ into $C(\mathrm{Q})$ yields 


$$
C\left(Q_{e e}^{*}\right)=\frac{(K+p f) \lambda}{\sqrt{\frac{2(K+p f) \lambda}{(h+p g)}}}+\lambda(c+p v)+\frac{(h+p g) \sqrt{\frac{2(K+p f) \lambda}{(h+p g)}}}{2}=\lambda(c+p v)+\sqrt{2(K+p f) \lambda(h+p g)}
$$

Note that $f, v$, and $g$ now directly denote the amount of emissions in the preceding analysis. Multiplying them with the tax rate $p$ again transforms the emissions into costs in monetary units. Incorporating a linear tax schedule is similar to the direct accounting of the environmental costs except for $f$ and $g$ being replaced by $p f$ and $p g$. When a sensitivity analysis of $Q_{e e}^{*}$ with respect to $Q^{*}$ is conducted, the same conditions as in the direct accounting setting presented in Proposition 1 (i) - (iii) may easily be obtained, independent of the tax rate $p$. In other words, the tax rate, $p$, in a linear tax schedule does not have an impact on the optimal policy (the order/production quantity in this case). However, it affects the total average cost as seen in $C\left(Q_{e e}^{*}\right)$ of Proposition 2. Hence, taxing carbon emissions gives incentive to identify emission sources, estimate the emission parameters and curb the emissions to achieve lower operating cost. Therefore, applying a carbon tax schedule suitable with the macroeconomic policy of a country is a useful tool for the regulatory agencies.

\subsubsection{Direct cap}

Letting $f, v$, and $g$ directly denote the emission amounts due to their respective activities is another approach, as applied in modeling the carbon tax setting. This approach also facilitates estimating the values of these environmental parameters. We also consider this approach in our analysis. For a more complete discussion on the sources of carbon emissions in inventory control, one can refer to [34-37].

The model in Eq. (3) and the preceding analysis in the direct accounting section ignore the total impact on environment caused by replenishment and inventory holding, since direct accounting for environmental costs does not give the organization an initiative to curb the emissions. Furthermore, not all organizations consider these costs willingly. This may not be true in case of a carbon tax imposed by the regulatory agencies where tax increases the monetary costs and the organizations are enforced to consider their environmental impact, as a consequence (see discussion on the carbon tax above).

An alternative modeling scheme is the one where a direct cap on environmental footprint is imposed either by the regulatory agencies or by the public awareness such that customers are seeking for more environmentally friendly products. In other words, the demand for the products may depend on the emission levels of the organization during the supply of its products to the customers.

Assume that there is an upper limit on the amount of GHG emissions, denoted by $\zeta$, as in the case of the countries signed the Kyoto Protocol [38]. Assume further that the above environmental cost parameters $(f, v, g)$ now denote the amount of emissions due to their respective activities mentioned before. Since the EOQ model is based on a single cycle and since the cycles are identical, $\zeta$ may also be assumed to be an upper bound on the average amount of GHG emissions per cycle (inducing a cap per unit product is another way of modeling, which would be used for gathering customer attention by carbon labeling the 
product; see [39-40]; see also Section $§ 2.2 .5$ for more details in carbon labeling). Therefore, the refined problem becomes

$$
\begin{gathered}
\text { Minimize } C(\mathrm{Q})=\frac{K \lambda}{Q}+\lambda c+\frac{h Q}{2} \\
\text { subject to } \\
\frac{f+v Q}{Q / \lambda}+g Q / 2 \leq \zeta \\
Q \geq 0
\end{gathered}
$$

This new model resembles the resource constrained EOQ model where a traditional case is to incorporate a linear constraint on the available warehouse space [25]. However in the above case, the constraint is nonlinear.

Proposition 3. Given a feasible solution to the model with a direct emissions cap, the optimal production lot size is

(i) $Q^{*}=\sqrt{\frac{2 K \lambda}{h}}$, if $\frac{f+v Q^{*}}{Q^{*} / \lambda}+g Q^{*} / 2 \leq \zeta$

(ii) $Q_{e e}^{*}=\frac{\zeta-\lambda v \pm \sqrt{(\lambda v-\zeta)^{2}-2 g \lambda f}}{g}$ otherwise, where $Q_{e e}^{*}$ satisfies $\frac{f \lambda}{Q_{e e}^{*}}+\lambda v+\frac{g Q_{e e}^{*}}{2}=\zeta$

Proof. (i) If $\frac{f+v Q^{*}}{Q^{*} / \lambda}+g Q^{*} / 2 \leq \zeta$, then $Q^{*}=\sqrt{\frac{2 K \lambda}{h}}$ is feasible. Since it is also optimal for the standard model, it is optimal for the above constrained nonlinear program. (ii) If $\frac{f+v Q^{*}}{Q^{*} / \lambda}+$ $g Q^{*} / 2>\zeta$, then the constraint in Eq. (6) is binding at the optimal solution. Therefore, the optimal production lot size, denoted by $Q_{e e}^{*}$, is obtained by solving the quadratic equation $\frac{f \lambda}{Q_{e e}^{*}}+\lambda v+\frac{g Q_{e e}^{*}}{2}=\zeta$ which yields $Q_{e e}^{*}=\frac{\zeta-\lambda v \pm \sqrt{(\lambda v-\zeta)^{2}-2 g \lambda f}}{g}$

Note that any order quantity should be a nonnegative real value to be valid. However, the quadratic equation in Proposition 3 (ii) may not have a real root or have two distinct or identical roots, depending on the parameters. If it has real roots, either one or both of the roots may be negative. Hence, the optimal policy is governed by the relationship among environmental and economic parameters of the organization.

Corollary 1. The emissions resulting from the direct cap model is

(i) $E M\left(Q^{*}\right)=\frac{f \lambda}{\sqrt{2 K \lambda / h}}+\lambda v+\frac{g \sqrt{2 K \lambda / h}}{2}$ if $\frac{f+v Q^{*}}{Q^{*} / \lambda}+g Q^{*} / 2 \leq \zeta$

(ii) $E M\left(\mathrm{Q}_{\mathrm{ee}}^{*}\right)=\zeta$, otherwise. 
Proof. (i) If $\frac{f+v Q^{*}}{Q^{*} / \lambda}+g Q^{*} / 2 \leq \zeta$, then the optimal policy of the direct accounting model is $Q^{*}=\sqrt{\frac{2 K \lambda}{h}}$ by Proposition 3. Plugging in $Q^{*}$ in the left-hand-side of Eq. (6) yields $E M\left(Q^{*}\right)=\frac{f \lambda}{\sqrt{2 K \lambda / h}}+\lambda v+\frac{g \sqrt{2 K \lambda / h}}{2}$. (ii) If $\frac{f+v Q^{*}}{Q^{*} / \lambda}+g Q^{*} / 2>\zeta$, then by Proposition 3 , the constraint is binding at optimality and the optimal policy satisfies $\frac{f \lambda}{Q_{e e}^{*}}+\lambda v+\frac{g Q_{e e}^{*}}{2}=\zeta=$ $\operatorname{EM}\left(Q_{e e}^{*}\right)$

Proposition 4. The optimal order/production quantity minimizing purely the emissions, $E M(Q)=\frac{f+v Q}{Q / \lambda}+g Q / 2$ is $Q_{e}^{*}=\sqrt{\frac{2 f \lambda}{g}}$.

Proof. Put $K=f$ and $h=g$ and by a similar derivation as in the standard model, $Q_{e}^{*}=$ $\sqrt{\frac{2 f \lambda}{g}} \operatorname{minimizes} \operatorname{EM}(Q)=\frac{f+v Q}{Q / \lambda}+g Q / 2$

\subsubsection{Cap \& Trade}

Another important mechanism to curb the emissions is the carbon trading markets, simply called as cap and trade. In this setting, companies emitting less than the allowed cap are rewarded whereas those over emitters are penalized. This penalty and reward mechanism is achieved via a carbon trading market. Companies emitting lower than the cap sell their allowances (the amount of emissions they are under the cap) whereas those emitting more than the cap buy such allowances. Therefore, the caps are not strict but encouraging in a cap and trade system. Such markets have already been developed in EU and US and the participation of the companies in the system is mandatory [41-42]. This market trades significant volumes now, and has a potential to grow up further [41].

Let the assumptions of the direct cap system hold along with the model parameters. Since the environmental parameters in the direct cap system denote the emission amounts due to their respective activities, we account for emissions directly in the cap and trade system as in the direct cap system. Assume further that $p$ now denotes the price of the carbon which is fixed and externally set by the market mechanism. Let $s^{+}$denote the amount of allowances sold by the organization and $s^{-}$denote the amount of allowances bought by the organization. Note that only one of these trading variables may be positive; i.e. the organization either buys or sells allowances. The optimal order/production quantity and the amount of allowances either sold or bought by the organization are found by solving the following mixed integer nonlinear program:

$$
\begin{gathered}
\text { Minimize } C(\mathrm{Q})=\frac{K \lambda}{Q}+\lambda c+\frac{h Q}{2}+p\left(s^{-}-s^{+}\right) \\
\text {subject to } \\
\frac{f+v Q}{Q / \lambda}+g Q / 2-s^{-}+s^{+} \leq \zeta
\end{gathered}
$$




$$
\begin{aligned}
& \mathrm{s}^{-} \leq \mathrm{y}_{1} M \\
& \mathrm{~s}^{+} \leq \mathrm{y}_{2} \zeta \\
& \mathrm{y}_{1}+\mathrm{y}_{2}=1 \\
& \mathrm{Q}, \mathrm{s}^{+}, \mathrm{s}^{-} \geq 0 \\
& \mathrm{y}_{1}, \mathrm{y}_{2} \in\{0,1\}
\end{aligned}
$$

where $M$ is a large positive number. The optimal cost and the emissions may also be obtained by solving the above model. Note that under the above model with a nonnegative carbon price, there are three options for the organization: (i) organization buys allowances if there is not a feasible $Q$ satisfying Eq. (6), (ii) neither buys nor sells allowances if there is a feasible $Q$ satisfying Eq. (6) at equality, (iii) and sells allowances if the constraint is satisfied but is not tight.

In this system, one important parameter is the carbon price, which appears to vary between 0 and 30 euro-cents per ton in the EU ETS [43]. This price is an exogenous system parameter determined by the market mechanism and assumed to be fixed in the above model. An alternative and intuitive scenario is the case where carbon price is dependent on the carbon cap. If the cap is tighter, the price of the allowance should obviously be higher. Therefore, one can assume an inversely proportional relation between $p$ and $\zeta$ as such:

$$
b=p+a \zeta
$$

where $a$ and $b$ are assumed to be nonnegative scalars without loss of generality. We can readjust the above model to incorporate such a relationship by plugging in $p=b-a \zeta$.

If regulatory agencies regulate the trading market by setting the carbon price, a macroeconomic view is needed as in the carbon tax model.

\subsubsection{Carbon Offsets}

The final environmental management mechanism we discuss is carbon offsets which stand for emission reducing investments. These investments may be in the form of energy efficient equipment and facilities, renewable energy resources, energy saving programs, carbon capturing and sequestration (CCS) systems, to name a few. The organization pays a price for the offset in return for reduced carbon footprint due to the increased technology and environmentally friendly resources.

Let the assumptions of the cap and trade system hold except for $p$ now denoting the unit price of the offsets and $s^{-}$denoting the amount of offset purchased by the organization. We assume that the offset directly relax the carbon emission constraint and does not reduce the values of emission parameters although this might be the case and modeled as well. The optimal order/production quantity and the amount of offset purchased may be found by solving the following nonlinear program:

$$
\begin{gathered}
\text { Minimize } C(\mathrm{Q})=\frac{K \lambda}{Q}+\lambda c+\frac{h Q}{2}+p s^{-} \\
\text {subject to } \\
\frac{f+v Q}{Q / \lambda}+g Q / 2-s^{-} \leq \zeta
\end{gathered}
$$




$$
Q, s^{-} \geq 0
$$

The optimal cost and emissions may also be obtained by solving the above nonlinear programming problem. However, it is important to note that buying offsets is reasonable only in the case that there is no feasible $Q$ satisfying Eq. (6). In such a case, it is mandatory (due to the cap exercised) to buy offsets to be able to maintain the operations.

The model for carbon offsets is similar to the model for cap and trade mechanism except for the allowances sold $\left(s^{+}\right)$in the cap and trade system. As in the cap and trade system, the emission amounts are directly accounted without converting them into monetary units. Moreover, a similar relationship between the offset price, $p$, and the carbon cap, $\zeta$, may also be considered as in the cap and trade system. Instead of a fixed price, $p$ may be inversely proportional to the carbon cap $\zeta$.

Purchasing offsets is optional for the organization although it is mandatory to participate in the cap and trade system. It may be the case that the market demands cleaner products with cleaner technology and energy. In such a case, carbon offsetting becomes obligatory in a sense for the organization to ensure competitive advantage. Nevertheless, this situation enables the organization to carbon-label its products and charge relatively larger prices to those environmentally sensitive customers. Let $r_{1}$ denote the price charged to usual customers and $r_{2}$ denote the price charged to environmentally sensitive customers when the organization purchases some offsets $\left(r_{2} \geq r_{1} \geq c\right)$. Then, the joint pricing and ordering/production model for the standard case is as follows:

$$
\begin{gathered}
\text { Minimize } C(\mathrm{Q})=\frac{K \lambda}{Q}+\lambda\left(c-r_{1}\right)+\frac{h Q}{2} \\
\text { subject to } \\
\frac{\mathrm{f}+\mathrm{vQ}}{\mathrm{Q} / \lambda}+\mathrm{g} / 2 \leq \mathrm{M} \\
\mathrm{Q} \geq 0
\end{gathered}
$$

where $M$ is a sufficiently large nonnegative carbon cap enabling a feasible $Q$ satisfying Eq. (20). On the other hand, the model with offsets can be formulated as follows:

$$
\begin{gathered}
\text { Minimize } C(\mathrm{Q})=\frac{K \lambda}{Q}+\lambda\left(c-r_{2}\right)+\frac{h Q}{2}+p s^{-} \\
\text {subject to } \\
\frac{f+v Q}{Q / \lambda}+g Q / 2-s^{-} \leq \zeta \\
Q, s^{-} \geq 0
\end{gathered}
$$

where $\zeta$ is sufficiently tighter which does not allow a feasible $Q$ satisfying Eq. (6).

A numerical comparison of the above two models (as can be seen in \$2.5.2) reveals the extent to which organizations may charge a relatively higher price for more environmentally friendly supply of products (via carbon offsets and tighter carbon caps) to those environmentally sensitive customers. This is not valid, of course, in markets where customers are non-sensitive to environmental friendliness. 


\subsection{EOQ Model with Social Criterion}

The corporate social responsibility (CSR) literature has been the primary area of investigation in terms of incorporating social criteria of sustainability. There is a vast body of literature on this subject; however, a literature review conducted in this area reveals that there are no studies concerned directly with the modeling of CSR aspects in supply chain and operations management problems in the open literature. When seeking appropriate supply chain metrics, SCOR model is the classical reference [44]. However, it does not provide any social metrics for supply chain modeling. The sustainable supply chain management literature also lacks social aspects as mentioned previously in the literature review section [11]. As a result, there is no straight forward metric available to use in modeling the social criteria. On the other hand, International Labor Organization [45] provides labor standards from which one can extract social metrics to be used in modeling the social criteria. Therefore, we rely on labor standards put forth by ILO.

According to ILO; there must be a legal upper limit on the working hours of employees [45-46]. On the other hand, the available man-hours is inevitably exhausted by the operations. Therefore, in order to assess the social performance of an organization, the amount of man-hours required to perform the operations can be used as a valid metric. Hence, we account for man-hours in modeling the social criterion. We employ direct accounting and direct cap approaches.

\subsubsection{Direct Accounting}

The following parameters are used: $m$ denote the fixed amount of man-hours required due to setups, order processing or transportation, $n$ denote the variable amount of man-hours required due to the production and related activities, and $l$ denote the man-hours required due to the inventory holding as a result of material handling and warehousing activities. By using the labor cost accounting, the cost of labor per man-hour can be easily obtained. Multiplication of the above man-hour requirement parameters with this cost factor yields the corresponding man-hour cost parameters of each activity. Let $m, n$, and $l$ also denote their respective cost correspondents. Assume further that the total available man-hours during a cycle is denoted by $W$.

Proposition 5. The optimal order/production quantity of the direct man-hours accounting model is $Q_{s e}^{*}=\sqrt{\frac{2(K+m) \lambda}{(h+l)}}$ and the corresponding optimal total cost is $C\left(Q_{s e}^{*}\right)=\lambda(c+n)+$ $\sqrt{2(K+m) \lambda(h+l)}$. Moreover, the following set of relationships holds:

(i) $Q_{s e}^{*}=Q^{*} \Leftrightarrow \frac{K}{h}=\frac{m}{l}$

(ii) $Q_{s e}^{*}>Q^{*} \Leftrightarrow \frac{K}{h}<\frac{m}{l}$

(iii) $Q_{s e}^{*}<Q^{*} \Leftrightarrow \frac{K}{h}>\frac{m}{l}$

Proof. Using a similar aggregation of costs as in deriving $Q_{e e}^{*}$, the proof of Proposition 5 is similar to the proof of Proposition 1; and therefore omitted for brevity. 
Apparently, the values of $m, n$, and $l$ rely on the abilities of the employees and the design of the work environment. One can argue that $n$ and $l$ are getting smaller due to automation in production environments whereas $m$ is most likely to be stable as a global trend. However, when they are assumed to represent their cost correspondents, they differ significantly between the developed and developing/under-developed countries. Hence, Proposition 5 (i)-(iii) explain why the under-developed countries exercise low quality mass production whereas the developed countries produce quality products in relatively higher lots. This also lays the foundation for mass customization in the developed countries where customization is achieved in a mass production setting.

\subsubsection{Direct Cap}

Alternatively, we may also employ the constrained-EOQ logic (since there is a legal upper limit on working hours and not all companies account for social costs willingly) and formulate the problem as follows:

$$
\begin{gathered}
\text { Minimize } C(\mathrm{Q})=\frac{K \lambda}{Q}+\lambda c+\frac{h Q}{2} \\
\text { subject to } \\
\frac{m+n Q}{Q / \lambda}+l Q / 2 \leq W \\
Q \geq 0
\end{gathered}
$$

Proposition 6. Given a feasible solution to the model with a direct man-hours cap, the optimal production lot size is

(i) $Q^{*}=\sqrt{\frac{2 K \lambda}{h}}$,if $\frac{m+n Q^{*}}{Q^{*} / \lambda}+l Q^{*} / 2 \leq W$

(ii) $Q_{s e}^{*}=\frac{W-\lambda n \pm \sqrt{(\lambda n-W)^{2}-2 l \lambda m}}{l}$ otherwise, where $Q_{s e}^{*}$ satisfies $\frac{m \lambda}{Q_{s e}^{*}}+\lambda n+\frac{l Q_{s e}^{*}}{2}=W$

Proof. Can be derived similarly as in the proof of Proposition 3.

The existence of a feasible $Q_{s e}^{*}$ depends on the values of the parameters of the above model as discussed previously for $Q_{e e}^{*}$.

Corollary 2. The required man-hours resulting from the direct man-hours cap model is

(i) $R M\left(Q^{*}\right)=\frac{m \lambda}{\sqrt{2 K \lambda / h}}+\lambda n+\frac{l \sqrt{2 K \lambda / h}}{2}$ if $\frac{m+n Q^{*}}{Q^{*} / \lambda}+l Q^{*} / 2 \leq W$

(ii) $R M\left(Q_{s e}^{*}\right)=W$, otherwise.

Proof. Follows from Proposition 6 and can be derived similarly as in the proof of Corollary 1. 
Proposition 7. The optimal order/production quantity minimizing purely the required manhours, $R M(Q)=\frac{f+v Q}{Q / \lambda}+g Q / 2$ is $Q_{s}^{*}=\sqrt{\frac{2 m \lambda}{l}}$.

Proof. Can be derived similarly as in the proof of Proposition 4.

\subsection{Triple Bottom Line Accounting}

In this part, we consider the case where the three pillars of sustainability are analyzed simultaneously. We model the three pillars using the direct accounting and direct cap modeling approaches, since these approaches are common for both the environmental and the social criteria. However, different modeling approaches may also be picked for environmental and social pillars (e.g. using cap and trade model for carbon footprint and direct cap modeling for man-hours) as well. We assume that the economic, environmental and social parameters are the same as in the previous sections.

\subsubsection{Direct Accounting}

By using the direct accounting approach, $Q_{e e s}^{*}$, the optimal order/production quantity with economic, environmental and social criteria can also be found similar to the previous subsections.

Proposition 8 . The optimal order/production quantity of the direct emissions and man-hours accounting model is $Q_{\text {ees }}^{*}=\sqrt{\frac{2(K+f+m) \lambda}{(h+g+l)}}$ and the corresponding optimal total cost is $C\left(Q_{\text {ees }}^{*}\right)=\lambda(c+v+n)+\sqrt{2(K+f+m) \lambda(h+g+l)}$. Moreover, the following set of relationships holds:

(i) $Q_{\text {ees }}^{*}=Q^{*} \Leftrightarrow \frac{K}{h}=\frac{f+m}{g+l}$

(ii) $Q_{\text {ees }}^{*}>Q^{*} \Leftrightarrow \frac{K}{h}<\frac{f+m}{g+l}$

(iii) $Q_{\text {ees }}^{*}<Q^{*} \Leftrightarrow \frac{K}{h}>\frac{f+m}{g+l}$

Proof. Can be derived similarly as in the proof of Proposition 1.

\subsubsection{Direct Cap}

The optimal order/production quantity with economic, environmental and social criteria may also be found using the direct cap modeling approach as the solution of the following nonlinear program: 


$$
\begin{aligned}
& \text { Minimize } C(Q)=\frac{K \lambda}{Q}+\lambda c+\frac{h Q}{2} \\
& \text { subject to } \\
& \frac{f+\mathrm{vQ}}{\mathrm{Q} / \lambda}+\mathrm{g} / / 2 \leq \zeta \\
& \frac{\mathrm{m}+\mathrm{nQ}}{\mathrm{Q} / \lambda}+\mathrm{l} / 2 \leq \mathrm{W} \\
& \mathrm{Q} \geq 0
\end{aligned}
$$

Proposition 9. Given a feasible solution to the model with a direct man-hours cap, the optimal production lot size is

(i) $Q^{*}=\sqrt{\frac{2 K \lambda}{h}}$,if $\frac{f+v Q^{*}}{Q^{*} / \lambda}+g Q^{*} / 2 \leq \zeta$ and $\frac{m+n Q^{*}}{Q^{*} / \lambda}+l Q^{*} / 2 \leq W$

(ii) $Q_{\text {ees }}^{*}=\frac{\zeta-\lambda v \pm \sqrt{(\lambda v-\zeta)^{2}-2 g \lambda f}}{g}$ where $Q_{\text {ees }}^{*}$ satisfies $\frac{f \lambda}{Q_{e e s}^{*}}+\lambda v+\frac{g Q_{e e s}^{*}}{2}=\zeta \quad$ and $\frac{m+n Q_{e e s}^{*}}{Q_{e e s}^{*} / \lambda}+l Q_{e e s}^{*} / 2 \leq W$

(iii) $Q_{e e s}^{*}=\frac{W-\lambda n \pm \sqrt{(\lambda n-W)^{2}-2 l \lambda m}}{l}$ where $Q_{e e s}^{*}$ satisfies $\frac{m \lambda}{Q_{e e s}^{*}}+\lambda n+\frac{l Q_{e e s}^{*}}{2}=W$ and $\frac{f+v Q_{e e s}^{*}}{Q_{e e s}^{*} / \lambda}+g Q_{e e s}^{*} / 2 \leq \zeta$

Proof. Can be derived similarly as in the proof of Proposition 3.

The existence of a feasible $Q_{e e s}^{*}$ depends on the values of the parameters of the above model as discussed previously for $Q_{e e}^{*}$ and $Q_{s e}^{*}$.

Corollary 3. The emissions and required man-hours resulting from the direct emissions and man-hours cap model is

(i) $E M\left(\mathrm{Q}^{*}\right)=\frac{f \lambda}{\sqrt{2 K \lambda / h}}+\lambda v+\frac{g \sqrt{2 K \lambda / h}}{2}$ and $R M\left(\mathrm{Q}^{*}\right)=\frac{m \lambda}{\sqrt{2 K \lambda / h}}+\lambda n+\frac{l \sqrt{2 K \lambda / h}}{2} \quad$ if $\frac{f+v Q^{*}}{Q^{*} / \lambda}+g Q^{*} / 2 \leq \zeta$ and $\frac{m+n Q^{*}}{Q^{*} / \lambda}+l Q^{*} / 2 \leq W$

(ii) $E M\left(Q_{\text {ees }}^{*}\right)=\zeta$ and $R M\left(Q_{\text {ees }}^{*}\right)=\frac{m+n Q_{e e s}^{*}}{Q_{e e s}^{*} / \lambda}+l Q_{e e s}^{*} / 2$ where $\frac{f \lambda}{Q_{e e s}^{*}}+\lambda v+\frac{g Q_{e e s}^{*}}{2}=\zeta$ and $\frac{m+n Q_{e e s}^{*}}{Q_{e e s}^{*} / \lambda}+l Q_{e e s}^{*} / 2 \leq W$

(iii) $R M\left(Q_{\text {ees }}^{*}\right)=W$ and $E M\left(Q_{\text {ees }}^{*}\right)=\frac{f+v Q_{e e s}^{*}}{Q_{e e s}^{*} / \lambda}+g Q_{e e s}^{*} / 2$ where $\frac{m \lambda}{Q_{e e s}^{*}}+\lambda n+\frac{Q_{e e s}^{*}}{2}=$ $W$ and $\frac{f+v Q_{e e s}^{*}}{Q_{e e s}^{*} / \lambda}+g Q_{e e s}^{*} / 2 \leq \zeta$. 
Proof. Follows from Proposition 6 and can be derived similarly as in the proof of Corollary 1.

\subsection{Numerical Analysis}

In this section, we present the analysis of a numerical study conducted for the sensitivity of the cap and trade and carbon offset models (presented in sections 2.2.4 and 2.2.5) with respect to changes in some model parameters. The solutions are obtained using default MINLP and NLP solvers of GAMS using default solver options [47].

Table 1 gives the base values of the model parameters used in the numerical experiments. The values in Table 2 are obtained via Eq. (2), Proposition 3, Corollary 1 and Proposition 4 using the data given in Table 1 and presented as a benchmark for sections 2.5.1 and 2.5.2.

Table 1. Parameter values for the base case in numerical analysis

\begin{tabular}{lll}
\hline Parameter & Units & Value \\
\hline$\lambda$ & Units & 50 \\
$K$ & $€ /$ setup & 40 \\
$C$ & $€ /$ unit & 12 \\
$H$ & $€ /$ unit-period & 2 \\
$F$ & $\mathrm{~kg} \mathrm{CO}_{2}$ & 60 \\
$V$ & $\mathrm{~kg} \mathrm{CO}_{2} /$ unit & 5 \\
$G$ & $\mathrm{~kg} \mathrm{CO}_{2} /$ unit & 1 \\
$\zeta$ & $\mathrm{kg} \mathrm{CO}_{2}$ & 300 \\
$P$ & $€ / \mathrm{unit}$ & 5 \\
$M$ & - & 1000000 \\
$A$ & - & 0.04 \\
$B$ & - & 20 \\
\hline
\end{tabular}

Table 2. Optimal solutions for the base case

\begin{tabular}{lcc}
\hline Item & \multicolumn{1}{l}{ Units } & Value \\
\hline EOQ & with & economic \\
$Q^{*}$ & units & 44.721 \\
$C\left(Q^{*}\right)$ & $€$ & 689.443 \\
$E M\left(Q^{*}\right)$ & $\mathrm{kg} \mathrm{CO}_{2}$ & 339.442 \\
\hline$E O Q$ with & environmental & objective only \\
$Q_{e}^{*}$ & units & 77.459 \\
$C\left(Q_{e}^{*}\right)$ & $€$ & 703.279 \\
$E M\left(Q_{e}^{*}\right)$ & $\mathrm{kg} \mathrm{CO}_{2}$ & 327.459
\end{tabular}


Table 2 shows that one should order/produce in the amount of 44.721 units if the objective is purely to minimize the economic costs with a total cost in the amount of $€ 689.443$. Furthermore, ordering/producing 44.721 units would lead to emissions in the amount of $339.442 \mathrm{~kg} \mathrm{CO}_{2}$. On the other hand, the order/production quantity would be in the amount of 77.459 units with a total cost of $€ 703.279$ if the objective is to minimize purely the emissions. With this environmental policy, the emission amount is reduced by $\% 3.5$ to an amount of $327.459 \mathrm{~kg} \mathrm{CO}_{2}$.

\subsubsection{Sensitivity of Parameters in the Cap \& Trade Model}

Figure 1 shows that organizations buy allowances whenever $p \geq 0$ and $\zeta<E M\left(Q_{e}^{*}\right)(\zeta=300)$. For $p=0$, the policy is reduced to ordering/producing $Q^{*}$, since buying allowances becomes cost-free in this extreme case. In other words, $p=0$ is equal to carbon cap constraint being inactive irrespective of the value of $\zeta$. As $p$ increases, the policy tends to move towards ordering/producing $Q_{e}^{*}$ with higher total cost while the amount of allowances bought is reduced gradually to the value of $E M\left(Q_{e}^{*}\right)-\zeta$. We assume that $p$ is externally set by the regulatory agencies or the market mechanism. In this case, all organizations decide and operate under the given price.

Figure 2 suggests that varying the carbon cap while carbon price is fixed $(p=5)$ does not have an impact on the optimal order/production quantity (conversely, varying the price affects the policy, as seen in Figure 1). Intuitively, one would expect that when $\zeta \mathrm{E} M\left(Q^{*}\right)$, the policy should be reduced to ordering/producing $Q^{*}$. However, for such values of $\zeta$, the organization makes money and reduces costs further by selling allowances in a cap and trade system, which is the main difference when compared to the carbon offset mechanism.

When the market price of the carbon is dependent on the cap $(20=p+0.04 \zeta)$ exercised by the regulatory agencies (or by the market mechanism like customer preferences), the effect of varying the carbon cap and price is experienced jointly. Figure 3 is similar to Figure 2 except for the policy being changed as well as higher costs being observed (due to higher carbon prices) for smaller values of $\zeta$. Note how $C(Q)$ diminishes as $\zeta$ increases. Organizations buy allowances until $\zeta$ is relaxed sufficiently (i.e. $\zeta \geq E M\left(Q_{e}^{*}\right)$ ) and sell allowances then onwards in a cap and trade system.

\subsubsection{Sensitivity of Parameters in the Carbon Offset Model}

Figure 4 is an exactly equivalent to Figure 1 except for the $s^{+}$data series, which is not valid for carbon offset model (since no carbon is sold). This shows that when $\zeta<E M\left(Q_{e}^{*}\right)$ $(\zeta=300)$, both systems respond to changes in $p$ in the same manner (although $p$ denotes carbon price in the cap and trade system whereas it denotes the offset price in the carbon offset mechanism). As price increases, the amount of offset purchased, $s^{-}$, decreases whereas order/production quantity increases (similar to the cap and trade system except for $s^{-}$denoting carbon allowances purchased in a cap and trade system).

Figure 5 is also similar to Figure 2 except for the $s^{+}$series, which is not valid for carbon offset model. This shows that when $\zeta<E M\left(Q_{e}^{*}\right)$, both systems respond to changes in $\zeta$ in the same manner given a fixed offset price, $p(p=5)$. However, when $\zeta E M\left(Q_{e}^{*}\right)$, no offsets are being purchased and the policy is gradually reduced to ordering/producing $Q^{*}$ as 
$\zeta E M\left(Q^{*}\right)$. Furthermore, $C\left(Q^{*}\right)$ becomes stable as $\zeta \geq E M\left(Q^{*}\right)$. Therefore, the offset system gives incentive to curb the emissions only if $\zeta<E M\left(Q_{e}^{*}\right)$.

When the offset price is dependent on the cap $(20=p+0.04 \zeta)$ exercised by the regulatory agencies (or by the market mechanism like customer preferences), the effects of varying the carbon cap and offset price changes are experienced jointly. Figure 6 is exactly equivalent to Figure 3 except for the $s^{+}$series in the offset system. As a result, the same policy with same cost structure is observed as in Figure 5 for $\zeta \geq E M\left(Q_{e}^{*}\right)$.

Finally, the extent to which organizations may carbon-label their products is tested. For $\zeta \geq E M\left(Q^{*}\right)$, there is no need for offsetting and hence carbon labeling is not valid for such caps. However, when the cap is tighter, then the price of the product should increase to achieve the cost value attained when $\zeta E M\left(Q^{*}\right)$ (compared with the case where $r_{1}=15$ and $p=5$ ). In order to lower the emissions by 10 units (i.e. $\zeta>329$ ); for example, the carbon labeling price $r_{2}$ should equal to 15.11 (see Figure 7).

\section{Insights}

In this section, we summarize the managerial insights based on the analysis given in previous sections.

\subsection{Incorporating sustainability into standard operational decision making processes has an impact on the operating policies of organizations.}

The analysis performed in this paper demonstrates how environmental and social concerns can be incorporated into a standard operational decision making model for inventory control. Since the optimal order/production quantity becomes a function of all the economic, social and environmental parameters, the cost structure is also affected by this revised model. Therefore, there are important differences in the policies and the resulting costs when the triple bottom line accounting of sustainability is considered.

\subsection{Organizations should estimate environmental and social parameters in order to achieve sustainability.}

Estimating environmental and social parameters is a necessity for the organizations to comply with the emerging legislative restrictions and market requirements. In order to operate sustainably, organizations should consider their environmental and social impacts in addition to economic factors. For this reason, managers need to identify the sources of these impact factors and estimate the parameters regarding these additional criteria in order to be able to model and assess environmental and social impacts of their organizations.

\subsection{Intervention by the regulatory agencies is the key to achieve sustainability until market awareness is established.}

Regulatory agencies should work to increase market awareness on decision making strategies that considers sustainability. Such a practice does not only favor customers but 
also the organizations and the whole economy in the long term. However, regulatory agencies should put in place tax schedules and caps until the natural market mechanism is established.

\subsection{Exercising caps on emissions (working hours) is the key to strictly curb emissions (working hours).}

The analysis show that mechanisms involving direct cost accounting and tax schedules give organizations incentive to investigate sources of environmental and social costs; however do not provide them with a rigid obligation to consider these added dimensions of sustainability. On the other hand, mechanisms involving a cap (direct cap, cap and trade, and carbon offset models) steer the organizations to adjust their policies accordingly. Therefore, a strict control of emissions (working hours) is possible only when caps are exercised by the regulatory agencies.

\subsection{Organizations should find ways to improve not only the economic cost parameters but also the environmental and social cost parameters.}

The analysis with the direct accounting approach shows that the revised optimal cost is always larger than the standard EOQ optimal even when the policy remains as the EOQ optimal order/production quantity. This suggests that organizations should improve their environmental and social impact parameters in addition to the economic ones. However, discussing these improvement opportunities is in the scope of another study, hence left aside.

\section{Conclusions}

Sustainability in supply chain management is an emerging requirement for a better business practice. In achieving sustainability, decision makers should adopt environmental and social considerations as well as the traditional economic objectives. This paper attempts to address the issue from an operations management perspective. We utilize a simple and widely used inventory control model; namely the EOQ model, and revise the standard model with additional environmental and social criteria. We provide alternative model formulations for a number of different settings and derive useful and practical results analytically and numerically for decision and policy makers.

This paper also serves as a reference point illustrating alternative environmental and social management approaches and their modeling apart from revising a classical model of an operational issue in supply chain management. As depicted, alternative modeling schemes are available for different approaches.

Further research directions are numerous. In our study, we consider the standard EOQ setting with a single item at a single location with no backlogging, constant lead times, and an unlimited supply. These assumptions may be relaxed to account for multiple items at multiple locations with planned backorders, variable lead times, and a finite production rate.

It is of course possible to include traditional extensions of EOQ model in the above models such as quantity discounts, imperfect quality, and resource constraints such as 
warehouse space. Furthermore, other forms of cost-accounting (such as NPV accounting instead of considering the average total cost) may also be utilized.

We consider a single organization in our study. Considering multiple organizations and echelons in the chain and analyzing the terms of coordination among supply chain members with these added environmental and social criteria will possibly reveal new insights for sustainable supply chain management. New types of contracts may also be designed which coordinates the chain sustainably. An interesting and possibly more complex problem in the cap and trade system; for instance, is the one where organizations decide on the price of the allowances. A proper treatment of such a problem would require a game theoretic analysis.

\section{References}

[1] Elkington J., Cannibals with forks: the triple bottom line of 21st century business, Capstone, Oxford, 2002.

[2] Corbett L.M., Sustainable operations management: a typological approach, Journal of Industrial Engineering and Management, 2, 1, 2009, 10-30.

[3] Corbett C.J., Klassen R.D., Extending the horizons: environmental excellence as key to improving operations, Manufacturing and Service Operations Management, 8, 1, 2006, $5-22$.

[4] Corbett C.J., Kleindorfer P.R., Environmental management and operations management: introduction to part 1 (manufacturing and eco-logistics), Production and Operations Management, 10, 2001a, 107-11.

[5] Corbett C.J., Kleindorfer P.R., Environmental management and operations management: introduction to part 2 (integrating operations and environmental management systems), Production and Operations Management, 10, 2001b, 225-27.

[6] Gupta M.C., Environmental management and its impact on the operations function, International Journal of Operations and Production Management, 15(8), 1995, 34-51.

[7] Gupta S.M., Lambert A.J.D., Environment conscious manufacturing. CRC Press, 2009.

[8] Kleindorfer P.R., Singhal K., Wassenhove L.N.V., Sustainable operations management, Production and Operations Management, 14, 4, 2005, 482-92.

[9] Linton J.D., Klassen R., Jayaraman V., Sustainable supply chains: an introduction, Journal of Operations Management, 25, 6, 2007, 1075-82.

[10] Sasikumar P., Kannan G., Issues in reverse supply chain, part III: classification and simple analysis, International Journal of Sustainable Engineering, 2, 2009, 2-27.

[11] Seuring S., Muller M., From a literature review to a conceptual framework for sustainable supply chain management, Journal of Cleaner Production, 16, 2008, 16991710 . 
[12] Srivastava S.K., Green supply-chain management: a state-of-the-art literature review, International Journal of Management Reviews, 9, 2007, 53-80.

[13] Bonney M., Jaber M., Environmentally responsible inventory models: non-classical models for a non-classical era, International Journal of Production Economics, 133, 1, 2011, 43- 53.

[14] Chen X., Benjaafar S., Elomri A., The carbon-constrained EOQ, Operations Research Letters, 41, 2, 2013, 172-179.

[15] Manikas A., Godfrey M., Inducing green behavior in a manufacturer, Global Journal of Business Research, 4, 2, 2010, 27-38.

[16] Letmathe P., Balakrishnan, N., Environmental considerations on the optimal product mix, European Journal of Operational Research, 167, 2005, 398-412.

[17] Penkuhn T., Spengler T., Pouchert H., Rentz O., Environmental integrated production planning for ammonia synthesis, European Journal of Operational Research, 97, 1997, 327-336.

[18] Bauer J., Bektaş T., Crainic T.G., Minimizing greenhouse gas emissions in intermodal freight transport: an application to rail service design, Journal of the Operational Research Society, 61, 3, 2009, 530-542.

[19] Kim N.S., Janic M., Wee B., Trade-off between carbon dioxide emissions and logistics costs based on multi objective optimization, Transportation Research Record, 2139, 2009, 107-116.

[20] Lee D., Dong M., Bian W., The design of sustainable logistics network under uncertainty, International Journal of Production Economics, 128, 2010, 159-66.

[21] Chaabane A., Ramudhin A., Paquet M., Design of sustainable supply chains under emission trading scheme, International Journal of Production Economics, 135, 1, 2011, 37-49.

[22] Neto J.Q.F., Bloemhof-Ruwaard, van Nunen J.A.E.E., van Heck E., Designing and evaluating sustainable logistics networks, International Journal of Production Economics, 111, 2008, 195-208.

[23] Erkut E., Karagiannidis A., Perkoulidis G., Tjandra S.A., A multicriteria facility location model for municipal solid waste management in North Greece, European Journal of Operational Research, 187, 2008, 1402-21.

[24] Zipkin P.H., Foundations of inventory management, McGraw Hill, 2000.

[25] Nahmias S., Production and operations analysis, McGraw Hill-Irwin, 1993.

[26] Harris F.W., How many parts to make at once?, Operations Research, 38, 6, 1990, 947-50. [Reprinted from Factory: The Magazine of Management, 10, 2, 1913, 135-136]. 
[27] Turkay M., Environmentally conscious supply chain management, in: Papageorgiou L., Georgiadis M. (eds.), Process systems engineering: supply chain optimization, WILEY-VCH, Weinheim, 2008, 45-86.

[28] Wiedmann T., Minx J., A definition of carbon footprint, in: Pertsova C.C. (ed.), Ecological economics research trends, Nova Science Publishers, Hauppauge NY, USA, 2008, 1-11.

[29] GHG Protocol, The Greenhouse Gas Protocol Initiative, http://www.ghgprotocol.org/ [accessed on 29/02/2012].

[30] ISO, International Organization for Standardization, http://www.iso.org/ [accessed on 29/02/2012].

[31] WRI, World Resource Institute, http://www.wri.org/ [accessed on 29/02/2012].

[32] EcoTransIT, http://www.ecotransit.org/ [accessed on 29/02/2012].

[33] Carbon Trust, http://www.carbontrust.co.uk/cut-carbon-reduce-costs/calculate/carbonfootprinting/pages/carbon-footprinting.aspx [accessed on 29/02/2012].

[34] Penman I., Stock J.R., Environmental issues in logistics, in: Robeson J.F., Copacino W.C. (eds.), The Logistics Handbook, The Free Press, New York, 1994, 840-57.

[35] Stock J.R., Reverse logistics, green logistics, and packaging, in: Taylor G.D. (ed.), Logistics engineering handbook. CRC, Boca Raton, 2008, 25-116.

[36] Stock J.R., Boyer S.L., Harmon T., Research opportunities in supply chain management, Journal of the Academy of Marketing Science, 38, 1, 2010, 32-41.

[37] Sundarakani B., de Souza R., Goh M., Modeling carbon footprints across the supply chain, International Journal of Production Economics, 128, 1, 2010, 43-50.

[38] UNFCCC, Kyoto Protocol, http://unfccc.int/kyoto protocol/items/2830.php [accessed on $29 / 02 / 2012]$.

[39] Brenton P., Edwards-Jones G., Jensena M.F., Carbon labeling and low income country exports: a review of the development issues, Development Policy Review, 27, 3, 2009, 243-267.

[40] Edwards-Jones G., Plassmann K., York E.H., Hounsome B., Jones D.L., Milà i Canals L., Vulnerability of exporting nations to the development of a carbon label in the United Kingdom, Environmental Science and Policy, 12, 2009, 479-90.

[41] EU European Union, Emissions Trading System, http://ec.europa.eu/clima/policies/ets/index_en.htm [accessed on 29/02/2012].

[42] European Commission, Questions and answers on emissions trading and national allocation plans, http://europa.eu/rapid/pressReleasesAction.do?reference=MEMO/08/35\&format=HTM L\&aged=0\&lang [accessed on 29/02/2012].

[43] European Carbon Exchange, http://www.ecx.eu [accessed on 29/02/2012]. 
[44] Supply-Chain Council, Supply-chain operations reference-model (SCOR), version 8.0, 2006.

[45] ILO, International Labor Organization, http://www.ilo.org [accessed on 29/02/2012].

[46] NZBCSD, New Zealand Business Council for Sustainable Development, Business guide to a sustainable supply chain: a practical guide. http://www.nzbcsd.org.nz/supplychain/ [accessed on 29/02/2012].

[47] GAMS, GAMSIDE build 2496/2589, License date Dec 24, 2007. Build VIS 22.6149.

Received October, 2013 


\section{Appendix A. Notation Used in Model Formulations}

$\lambda$

time index

$I(t) \quad$ inventory level at time $t$

demand rate

$T \quad$ cycle length

K fixed/setup cost

c variable cost

h holding cost

$f \quad$ fixed amount of $G H G$ emissions

$v \quad$ variable amount of $G H G$ emissions

$g$

amount of $G H G$ emissions due to inventory holding

$m \quad$ fixed amount of man-hours required

$n \quad$ variable amount of man-hours required

$l \quad$ man-hours required due to inventory holding

$r_{1} \quad$ product price charged to regular customers

$r_{2} \quad$ product price charged to environmentally sensitive customers

$p \quad$ tax rate/carbon price/offset price

$\zeta \quad$ GHG emissions cap

available amount of man-hours/working hour limit

a nonnegative scalar

a nonnegative scalar

$b$

a large nonnegative scalar

amount of allowances sold

amount of allowances/offsets purchased

a binary decision variable

$y_{1}$

a binary decision variable

$Q$

order/production quantity

$Q^{*}$

optimal order/production quantity in the standard EOQ model

$Q_{e}^{*}$

optimal order/production quantity with pure environmental criterion

$Q_{s}^{*}$

$Q_{e e}^{*}$ optimal order/production quantity with pure social criterion

$Q_{s e}^{*}$ optimal order/production quantity with economic and environmental criteria

$Q_{\text {ees }}^{*}$ optimal order/production quantity with social and economic criteria

$C(Q) \quad$ total average cost of replenishment

$C^{\prime}(Q) \quad$ first derivative of $C(Q)$ with respect to $Q$ 
$C^{\prime \prime}(Q) \quad$ second derivative of $C(Q)$ with respect to $Q$

$C\left(Q^{*}\right) \quad$ optimal total average cost with standard EOQ optimal

$C\left(Q_{e}^{*}\right) \quad$ optimal total average cost with pure environmental criterion

$C\left(Q_{e e}^{*}\right) \quad$ optimal total average cost with economic and environmental criteria

$C\left(Q_{s e}^{*}\right) \quad$ optimal total average cost with social and economic criteria

$C\left(Q_{\text {ees }}^{*}\right) \quad$ optimal total average cost with triple bottom line accounting

$E M\left(Q^{*}\right) \quad$ optimal total average emissions with standard EOQ optimal

$E M\left(Q_{e}^{*}\right) \quad$ optimal total average emissions with pure environmental criterion

$E M\left(Q_{e e}^{*}\right) \quad$ optimal total average emissions with economic and environmental criteria

$R M\left(Q^{*}\right) \quad$ optimal total average man-hours required with standard EOQ optimal

$R M\left(Q_{s e}^{*}\right) \quad$ optimal total average man-hours required with social and economic criteria

\section{Appendix B. Figures}

Cap \& Trade - Varying Carbon Price

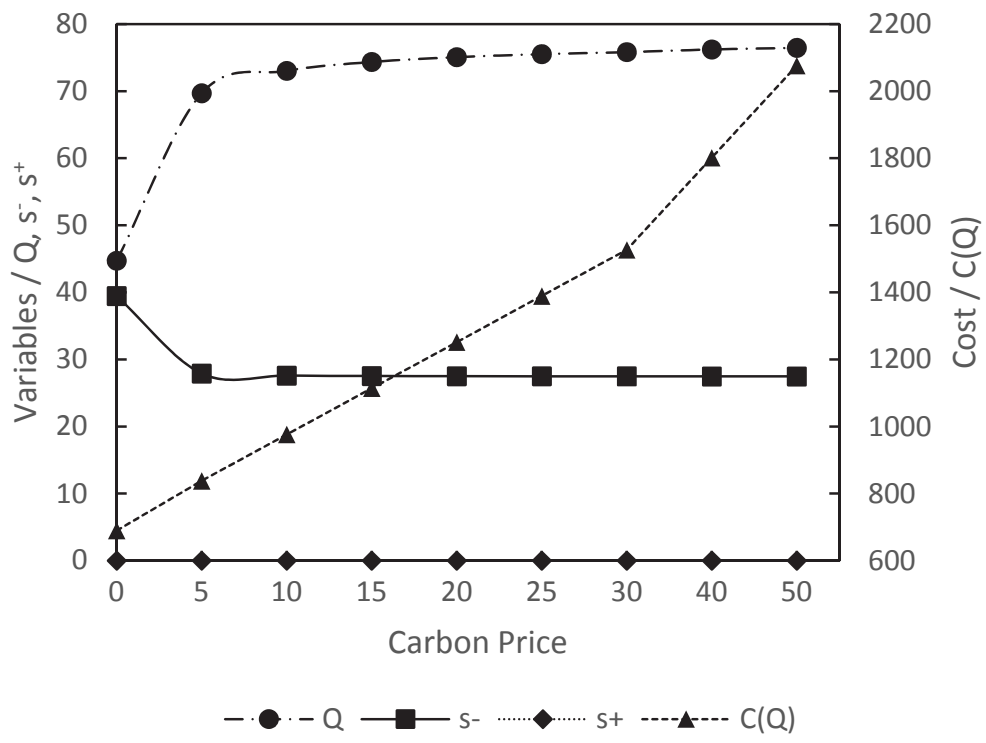

Figure 1. Varying carbon price 
Cap \& Trade - Varying Carbon Cap

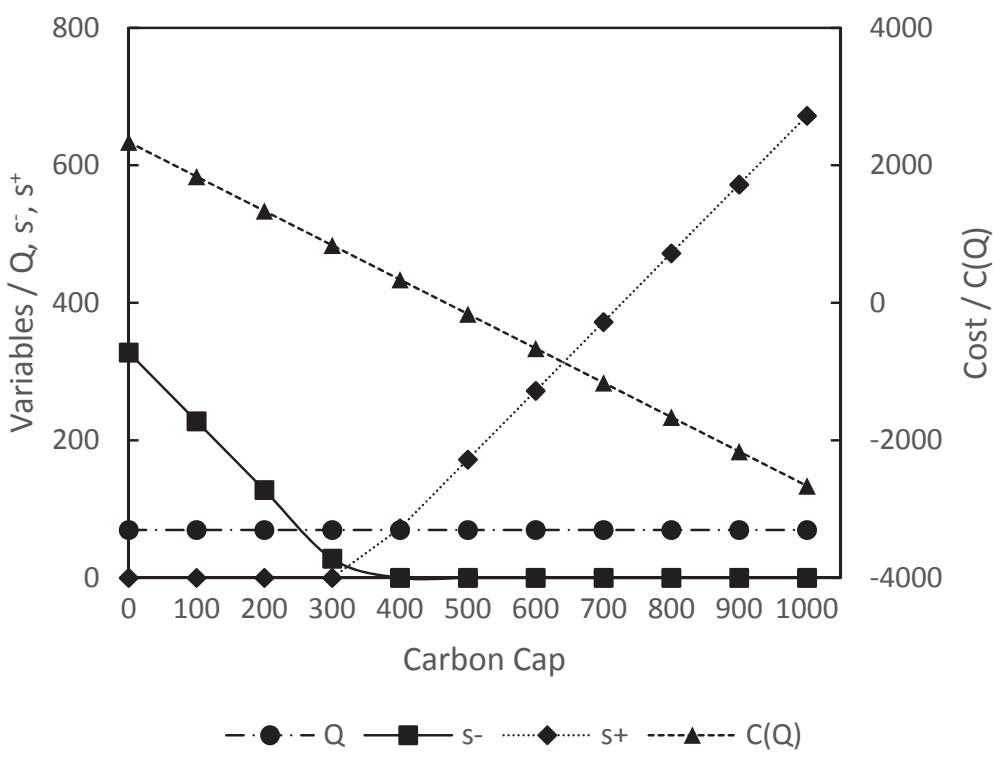

Figure 2. Varying carbon cap

Cap-dependent Carbon Price - Varying Carbon Cap

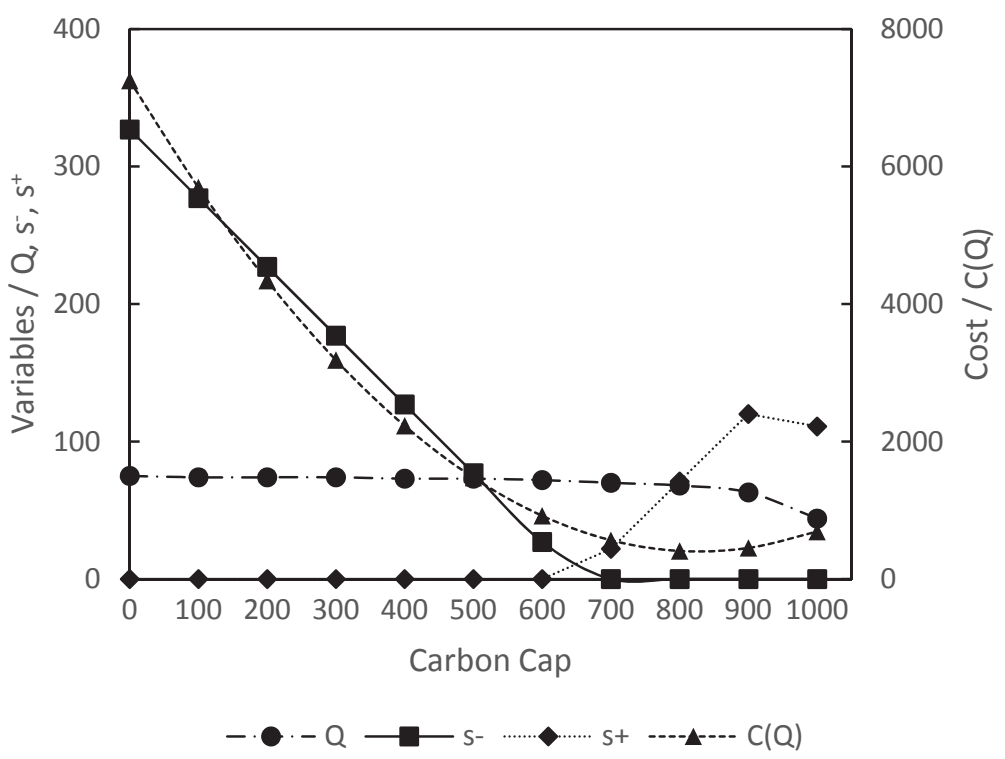

Figure 3. Cap-dependent carbon price 


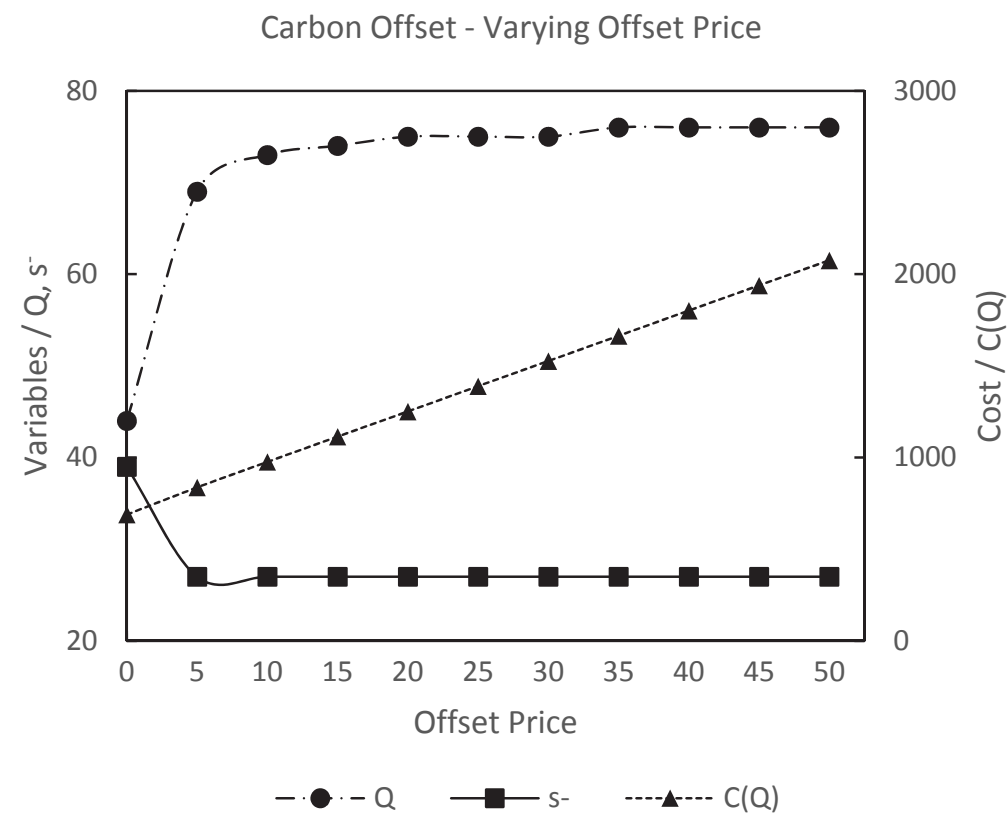

Figure 4. Varying offset price

Carbon Offset - Varying Carbon Cap

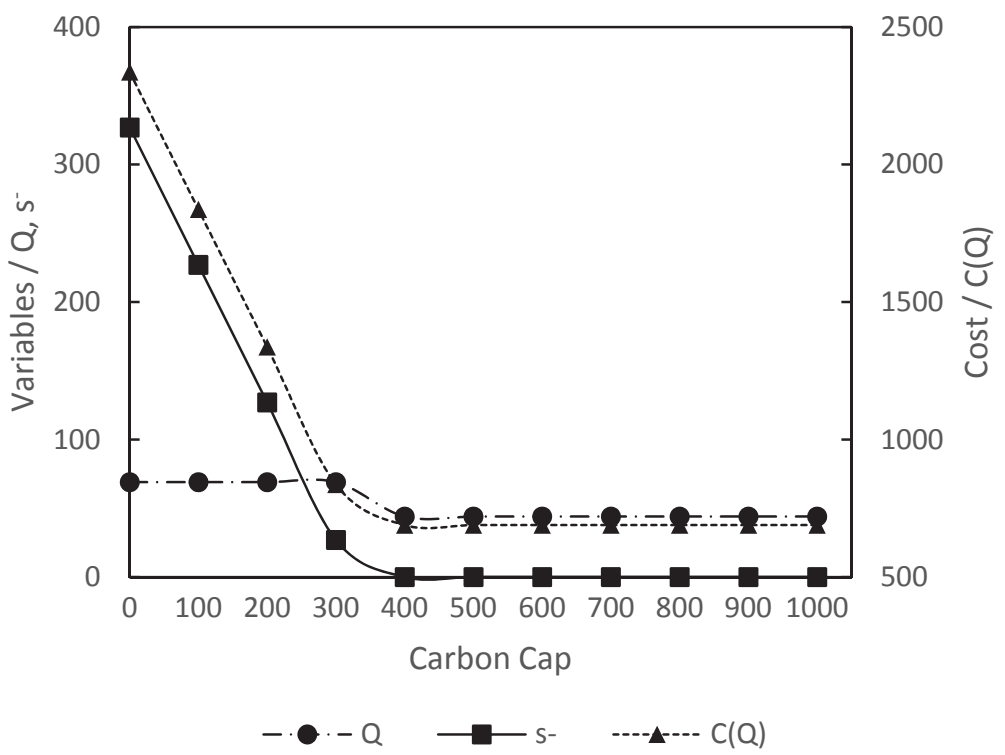

Figure 5. Varying carbon cap 
Cap-dependent Offset Price - Varying Carbon Cap

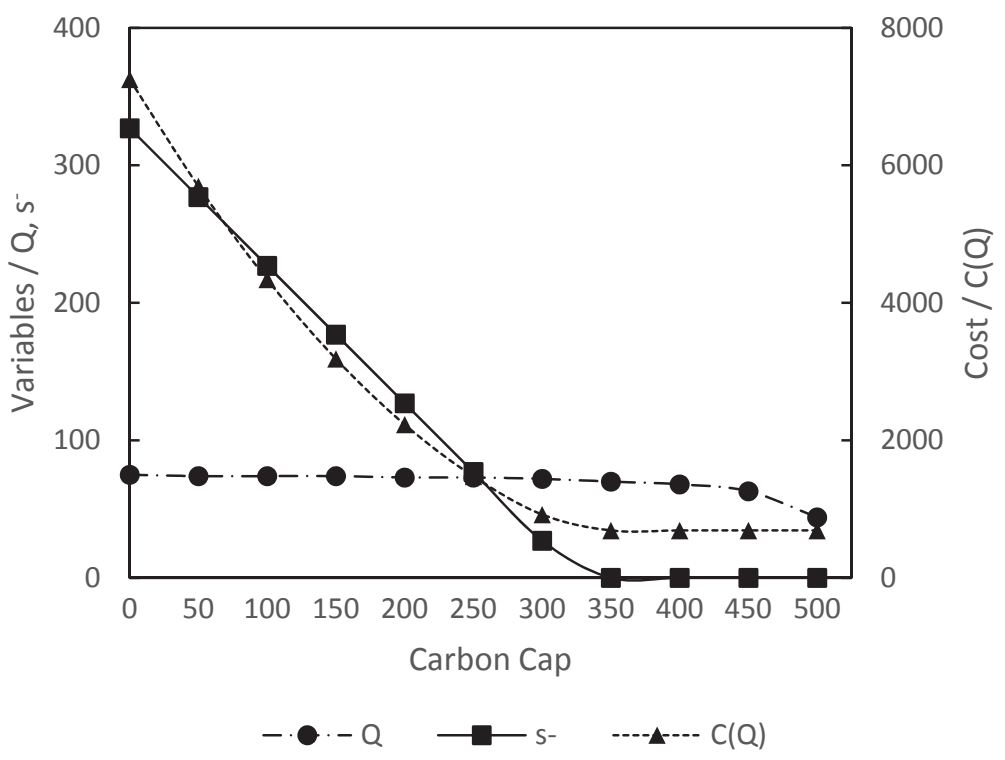

Figure 6. Cap-dependent offset price

Carbon Labeling - Varying Carbon Cap

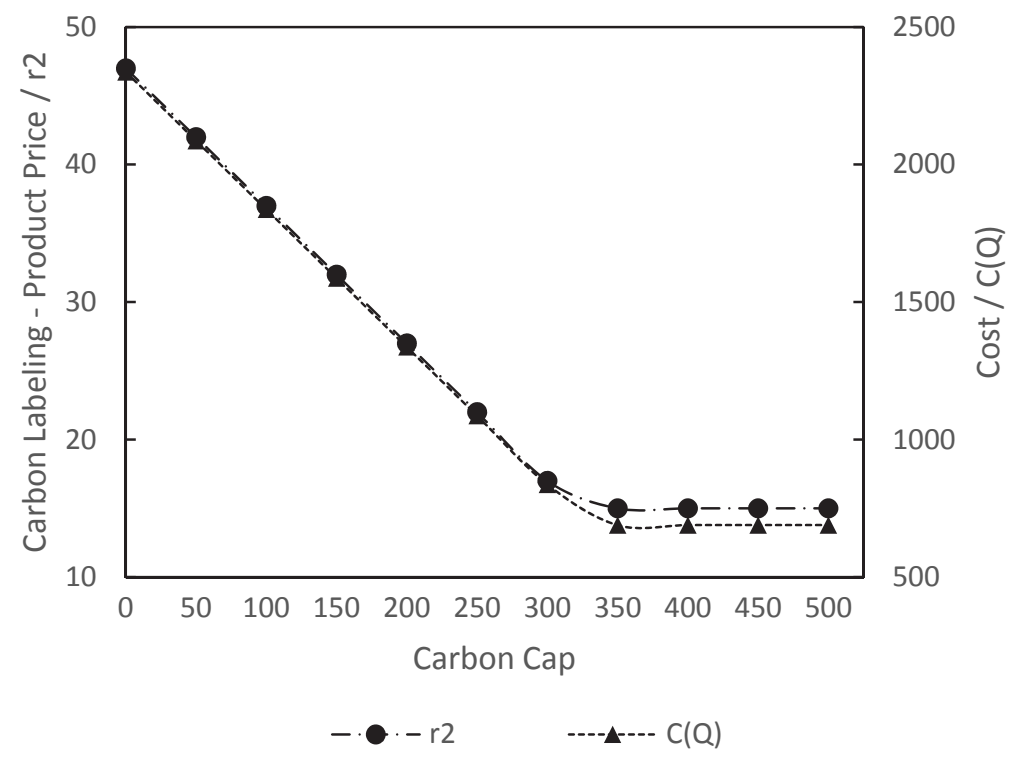

Figure 7. Carbon Labeling 